\title{
CORRECTION
}

\section{Correction to: The offline data quality monitoring of the BESIII end-cap TOF system}

Ming-Ming Ma1,2 $\cdot$ Jing-Yi Liu' ${ }^{1,2}\left(\mathbb{0} \cdot\right.$ Shuo-Pin Wen ${ }^{1} \cdot$ Sheng-Sen Sun ${ }^{1,2} \cdot$ Chun-Xiu Liu ${ }^{1} \cdot$ Yong-Zhao Sun ${ }^{1}$ Zi-Yan Deng ${ }^{1} \cdot$ Ye Yuan ${ }^{1} \cdot$ Hong-Liang Dai ${ }^{1,3} \cdot$ Zhi Wu$^{1,3} \cdot$ Yue-Kun Heng ${ }^{1,2,3}$. Huai-Min Liu ${ }^{1,2,3}$

Published online: 23 December 2021

(c) Institute of High Energy Physics, Chinese Academy of Sciences 2021

\section{Correction to: \\ Radiation Detection Technology and Methods (2019) 3:58 \\ https://doi.org/10.1007/s41605-019-0132-0; (2021) \\ 5:207-212 https://doi.org/10.1007/s41605-021-00251-y}

The articles https://doi.org/10.1007/s41605-019-0132-0, https://doi.org/10.1007/s41605-021-00251-y have been inadvertently got published twice and were included in Volume 3 Issue 4 (2019), Volume 5 issue 2 (2021)

Both articles are similar and the Publisher apologizes to the authors and readers for the inconvenience caused by this error.

The original articles can be found online at https://doi.org/10.1007/ s41605-019-0132-0 and https://doi.org/10.1007/s41605-021-00251-y.

$\triangle$ Jing-Yi Liu

liujy@ihep.ac.cn

1 Institute of High Energy Physics, Chinese Academy of Sciences, Beijing 100049, China

2 University of Chinese Academy of Sciences, Beijing 100049, China

3 State Key Laboratory of Particle Detection and Electronics, Beijing 100049, China 